\title{
A lognormal type stochastic volatility model with quadratic drift*
}

\author{
Peter Carr ${ }^{\dagger}$ and Sander Willems ${ }^{\ddagger}$
}

July 17, 2019

\begin{abstract}
This paper presents a novel one-factor stochastic volatility model where the instantaneous volatility of the asset log-return is a diffusion with a quadratic drift and a linear dispersion function. The instantaneous volatility mean reverts around a constant level, with a speed of mean reversion that is affine in the instantaneous volatility level. The steady-state distribution of the instantaneous volatility belongs to the class of Generalized Inverse Gaussian distributions. We show that the quadratic term in the drift is crucial to avoid moment explosions and to preserve the martingale property of the stock price process. Using a conveniently chosen change of measure, we relate the model to the class of polynomial diffusions. This remarkable relation allows us to develop a highly accurate option price approximation technique based on orthogonal polynomial expansions.
\end{abstract}

\section{Introduction}

The popularity of the Heston (1993) model and, more generally, affine models (see e.g., Duffie et al. (2003)) for modeling stochastic volatility is in large part due to their analytical tractability. However, there is abundant empirical evidence that favours non-affine models, in particular specifications with a lognormal type of diffusion for the (instantaneous) volatility 11 For instance, Christoffersen et al. (2010) show that absolute changes in realized volatility are positively correlated with the volatility level and do not follow a Gaussian distribution. In contrast, the Heston model implies that (instantaneous) changes in volatility should be Gaussian and independent of the volatility level. Changes in the log realized volatility, on the other hand, closely resemble a normal distribution, which motivates the use of a lognormal type of diffusion component in the volatility process 2 Figure 1 reproduces these results using a 5-minute sub-sampled daily realized volatility measure for the S\&P500 index from January 2000 until June 2019 and confirms the findings of Christoffersen et al. (2010).

Lognormal type stochastic volatility models are, however, particularly prone to problems such as moment explosions and loss of the martingale property for the asset price, see e.g. Lions and Musiela (2007) and Andersen and Piterbarg (2007). These problems are caused by the fat right tail of the volatility distribution, which can cause large spikes in the asset price. Having finite higher order

\footnotetext{
*We thank Damien Ackerer for helpful comments.

${ }^{\dagger}$ New York University, Tandon School of Engineering

${ }^{\ddagger}$ École Polytechnique Fédérale de Lausanne (EPFL) and Swiss Finance Institute

${ }^{1}$ With lognormal type of diffusion we mean a diffusion $\sigma_{t}$ with $\mathrm{d}[\sigma, \sigma]_{t}=\nu^{2} \sigma_{t}^{2} \mathrm{~d} t$, for some $\nu>0$.

${ }^{2}$ See Christoffersen et al. (2010) for the S\&P500 index, Andersen et al. (2001) for individual stocks in the DJIA index, and Andersen et al. (2001) for foreign exchange markets.
} 
moments for the asset price is important, for example, to price derivatives with a super-linear payoff. Andersen and Piterbarg (2007) highlight the importance of these type of derivatives in interest rate markets. Moreover, when pricing derivatives with Monte-Carlo simulations, the payoff needs to have a finite second moment in order to use the central limit theorem to derive confidence intervals on the Monte-Carlo estimators. For example, if the volatility process has an affine drift and a linear dispersion function, then the instantaneous correlation between log-price and volatility has to be smaller than $-87 \%$ in order for the asset price to have a finite fourth moment. In this paper, we propose a novel non-affine one-factor stochastic volatility model featuring a diffusion with a quadratic drift function and a linear dispersion function for the volatility process. The quadratic term has a negative coefficient in our model, which allows for a rapid reduction following an upward spike in the volatility 3 The linear dispersion function produces lognormal type innovations in the volatility and the quadratic term in the drift controls undesirable side effects such as moment explosions and loss of martingality. Moreover, using the critical moment formula of Lee (2004), we show that a nonzero quadratic term in the drift allows to control both the small strike and large strike tail of the Black-Scholes implied volatility skew. The volatility process in our model is stationary and has a Generalized Inverse Gaussian (GIG) distribution as steady-state distribution. The GIG distribution, which contains the inverse Gaussian, hyperbolic, gamma, and inverse-gamma as special cases, has broad empirical support for modeling stochastic volatility in stock returns, see for example Barndorff-Nielsen (1997), Eberlein (2001), Eberlein and Prause (2002), and Gander and Stephens (2007).

Since our model is far from affine, tractability is not straightforward. If we set the quadratic term in the drift of the volatility to zero, then our model fits in the class of polynomial diffusions, see e.g. Filipović and Larsson (2016). This class of stochastic processes, which contains all affine diffusions as special cases, is characterized by the fact that their infinitesimal generator maps polynomials to polynomials of the same degree or less. As a consequence, all conditional moments of the log-asset price are available in closed form and European style derivatives on the asset price can be priced using moment-based approximation methods, see e.g. Ackerer and Filipović (2019) 4 The polynomial property is lost, however, as soon as we have a nonzero quadratic term in the drift of the volatility. We circumvent this problem by introducing a change of measure under which the polynomial property is recovered. Under the new measure, derivative prices are given by the expectation of the discounted payoff multiplied by the Radon-Nikodym density of the measure change. We show how to compute all joint conditional moments of the log-asset price and log-Radon-Nikodym density in closed form under the new measure. An orthogonal polynomial expansion technique in the spirit of Ackerer and Filipovic (2019) then allows us to efficiently price European style derivatives on the asset price.

The remaining of this paper is structured as follows. Section 2 describes the model dynamics and Section 3 analyzes the steady-state distribution of the volatility process. In Section 4 we study the problem of moment explosions. Section 5 relates our model to the class of polynomial diffusions, which is used in Section 6 to develop a derivative pricing approximation method. Section 7 contains a numerical study of the model and Section 8 concludes. All proofs and additional technical results are collected in the Appendix.

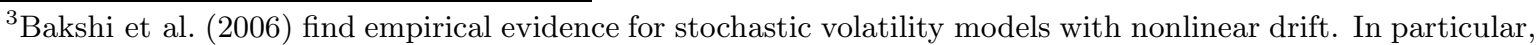
they find a significantly negative coefficient on the quadratic term in the drift.

${ }^{4}$ For applications of polynomial processes in derivative pricing, see for example Filipović et al. (2016), Ackerer and Filipović (2016), Filipović and Willems (2017), Ackerer et al. (2018), and Willems (2019).
} 


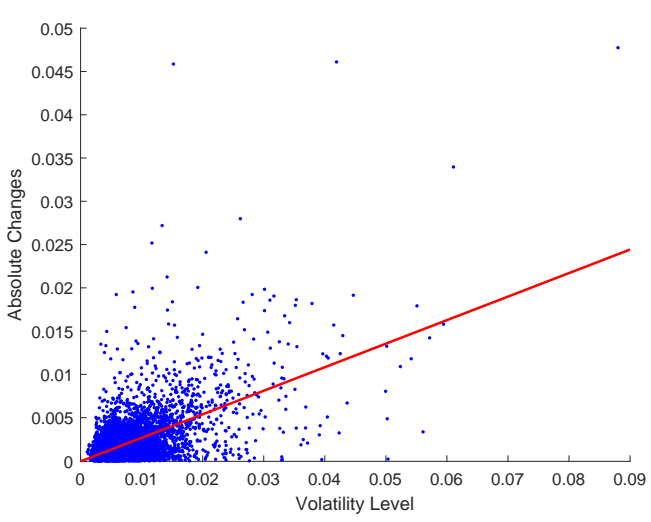

(a) Absolute change in volatility

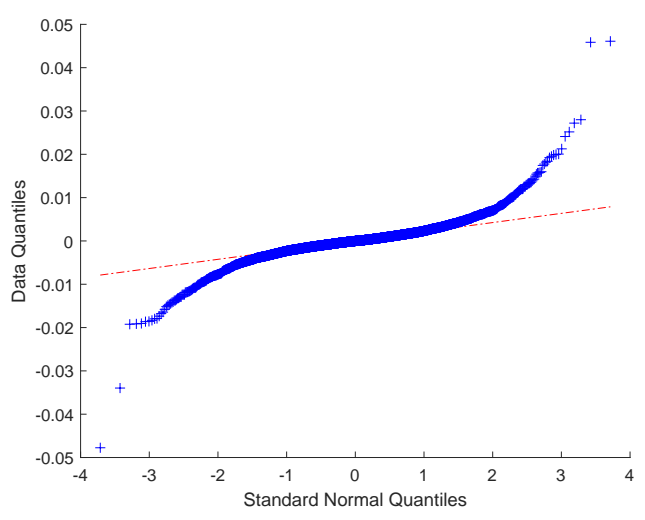

(c) Changes in volatility QQ-plot

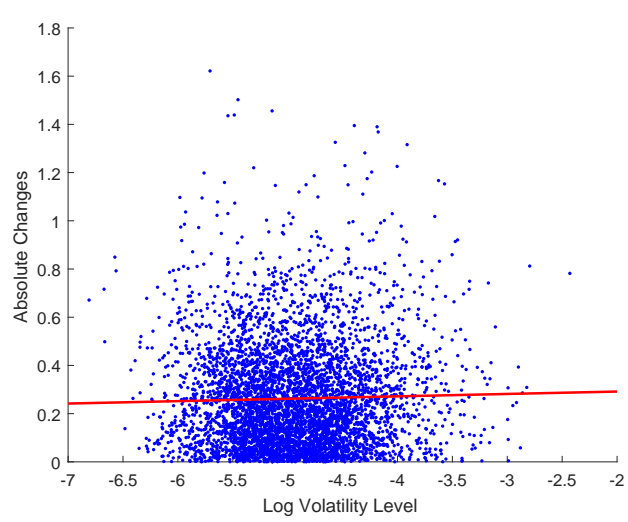

(b) Absolute change in log volatility

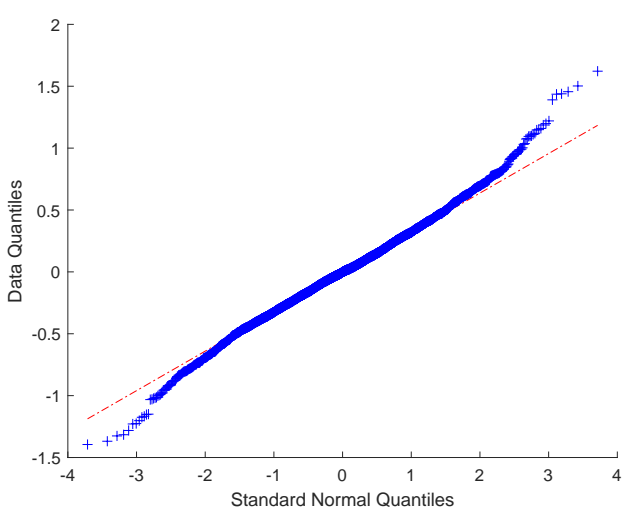

(d) Changes in log volatility QQ-plot

Figure 1: The top left (right) figure shows a scatter plot of the realized (log) volatility level against the absolute change one day ahead, together with a least-squares regression line in red. The bottom left (right) figure shows a quantile-quantile plot of daily changes in realized (log) volatility. Realized volatilities are obtained from Oxford-Man Institute's realized library using 5 -minute sub-sampled high-frequency returns on the S\&P500 index from January 2000 until June 2019.

\section{Model specification}

We consider a financial market modeled on a filtered probability space $\left(\Omega, \mathcal{F}, \mathcal{F}_{t}, \mathbb{Q}\right)$, where $\mathbb{Q}$ is a risk-neutral probability measure. Henceforth $\mathbb{E}_{t}[\cdot]$ denotes the $\mathcal{F}_{t}$-conditional $\mathbb{Q}$-expectation. Let $S_{t}$ denote the stock price and assume for simplicity zero interest rates and no dividend payments 5 We specify the following $\mathbb{Q}$-dynamics for the $\log$-price $x_{t}=\log \left(S_{t}\right)$

$$
\begin{aligned}
& \mathrm{d} x_{t}=-\frac{1}{2} \sigma_{t}^{2} \mathrm{~d} t+\sigma_{t}\left(\rho \mathrm{d} W_{t}+\sqrt{1-\rho^{2}} \mathrm{~d} B_{t}\right), \\
& \mathrm{d} \sigma_{t}=\left(R_{0}+R_{1} \sigma_{t}\right)\left(R_{2}-\sigma_{t}\right) \mathrm{d} t+\nu \sigma_{t} \mathrm{~d} W_{t},
\end{aligned}
$$

\footnotetext{
${ }^{5}$ Alternatively, one can also think of $S_{t}$ as, for example, an interest rate variable (e.g., forward rate or swap rate) and replace $\mathbb{Q}$ by the appropriate pricing measure.
} 
with $R_{0}, R_{1} \geq 0, R_{2}, \nu, \sigma_{0}>0, \rho \in[-1,1], x_{0} \in \mathbb{R}$, and $W_{t}, B_{t}$ independent $\mathbb{Q}$-Brownian motions. The volatility process mean-reverts around a constant level $R_{2}$ with a stochastic speed of mean-reversion $R_{0}+R_{1} \sigma_{t}$. Our model specification nests many existing models. The lognormal SABR model of Hagan et al. (2002) arises when $R_{0}=R_{1}=0$, in which case $\sigma_{t}$ is simply a geometric Brownian motion without drift. In this case, however, the volatility process is not mean-reverting, which is an important empirical feature. If we set $R_{0}>0$ and $R_{1}=0$, then $\sigma_{t}$ becomes a mean-reverting diffusion with affine drift and linear dispersion function, which we refer to as a linear diffusion. This type of model has been studied in Lewis $(2000)$, Karasinski and Sepp (2012), Sepp (2014, 2016), Lee et al. (2016), and Ackerer and Filipović (2019) 6 For $R_{0}=0$ and $R_{1}>0$, equation (2) is known as the logistic diffusion and originated in the context of modeling constrained population growth in biology, see e.g. Tuckwell and Koziol (1987).7 In the context of finance, the logistic diffusion has been used, for example, in a general equilibrium model by Merton (1975) and in a stochastic volatility model by Hull and White (1987) and Lewis (2019) 8

The following proposition shows that the model is well defined and that zero is an unattainable boundary for $\sigma_{t}$.

Proposition 2.1. There exists a unique strong solution $\left(x_{t}, \sigma_{t}\right)$ of (11)-(2) taking values in $\mathbb{R} \times(0, \infty)$.

From the proof of Proposition 2.1, it becomes clear that the non-negativity assumption $R_{1} \geq 0$ is crucial in order for (2) to have a global solution, cf. Remark A.1. Indeed, if $R_{1}<0$, then $\sigma_{t}$ blows up in finite time.

Remark 2.2. Although zero is a natural lower bound for the volatility process, we can generalize (2) by adding a lower bound $\underline{\sigma} \geq 0$ as follows

$$
\mathrm{d} \sigma_{t}=\left(R_{0}+R_{1}\left(\sigma_{t}-\underline{\sigma}\right)\right)\left(R_{2}-\left(\sigma_{t}-\underline{\sigma}\right)\right) \mathrm{d} t+\nu\left(\sigma_{t}-\underline{\sigma}\right) \mathrm{d} W_{t},
$$

with $\sigma_{0}>\underline{\sigma}$ and the same restrictions on the other parameters as before. All the results we derive in our paper are easily adjusted to accommodate this generalization.

\section{Steady-state distribution of $\sigma_{t}$}

In this following proposition, we explicitly derive the steady-state distribution of $\sigma_{t}$.

Proposition 3.1. If either $R_{0}>0$, or $R_{0}=0$ and $2 R_{1} R_{2}>\nu^{2}$, then the process $\sigma_{t}$ has a steady-state distribution with density function

$$
\pi(x) \propto x^{\xi-1} \exp \left\{-2 \frac{R_{0} R_{2}}{\nu^{2}} \frac{1}{x}-2 \frac{R_{1}}{\nu^{2}} x\right\},
$$

where $\xi=-2 \frac{R_{0}-R_{1} R_{2}}{\nu^{2}}-1$. If $R_{0}=0$ and $2 R_{1} R_{2} \leq \nu^{2}$, then $\sigma_{t} \rightarrow 0$ a.s. as $t \rightarrow \infty$.

\footnotetext{
${ }^{6}$ Also related is the GARCH diffusion model of Nelson (1990) and Barone-Adesi et al. (2005), where $\sigma_{t}^{2}$ is modeled as a diffusion with affine drift and linear dispersion function. Applying Itô's lemma shows that the corresponding volatility process also has a linear dispersion function, but it does not have an affine drift. Directly modeling volatility seems more intuitive and provides a more natural interpretation for the model parameters.

${ }^{7}$ The deterministic version of this SDE was developed in the early 19th century by the Belgian mathematician Pierre Frangis Verhulst to model population growth.

8 Hull and White (1987) specify $\mathrm{d} \sigma_{t}^{2}=a\left(\sigma^{*}-\sigma_{t}\right) \sigma_{t}^{2} \mathrm{~d} t+\xi \sigma_{t}^{2} \mathrm{~d} W_{t}$ for some parameters $a, \sigma^{*}, \xi>0$. Applying Itô's lemma shows that $\sigma_{t}$ follows a logistic diffusion: $\mathrm{d} \sigma_{t}=\frac{a}{2} \sigma_{t}\left(\sigma^{*}-\frac{\xi^{2}}{4}-\sigma_{t}\right) \mathrm{d} t+\frac{\xi}{2} \sigma_{t} \mathrm{~d} W_{t}$.
} 
The steady-state distribution therefore belongs to the class of Generalized Inverse Gaussian distributions, see e.g. Jorgensen (1982). The integration constant such that $\pi$ integrates to one is provided in the proof (see Section A.2). We distinguish four different cases, based on the values for $R_{0}$ and $R_{1}$ :

1. For $R_{0}=R_{1}=0$, the process $\sigma_{t}$ becomes a geometric Brownian motion without drift, which goes to zero almost surely as $t \rightarrow \infty$.

2. For $R_{0}>0$ and $R_{1}=0$, the process $\sigma_{t}$ becomes a linear diffusion and we recover the inverse gamma distribution as steady-state distribution, see e.g. Barone-Adesi et al. (2005). The first moment of $\pi$ is equal to $R_{2}$, so that $R_{2}$ can be interpreted as the long-term level of mean reversion. Higher order moments do not always exist because the inverse gamma distribution has a right tail with polynomial decay. Remark that $\pi(0)=0$, regardless of $R_{0}$ and $R_{2}$, due to the exponential decay of of the left tail.

3. For $R_{0}=0$ and $R_{1}>0$, the process $\sigma_{t}$ becomes a logistic diffusion. If $2 R_{1} R_{2} \leq \nu^{2}$, then a similar behavior as in the first case occurs and $\sigma_{t} \rightarrow 0$ almost surely as $t \rightarrow \infty$. If $2 R_{1} R_{2}>\nu^{2}$, we recover the gamma distribution as steady-state distribution, which has finite moments of any order. In particular, the first moment equals $R_{2}-\frac{\nu^{2}}{2 R_{1}}$. As highlighted in Merton (1975) and Ewald and Yang (2007), we can therefore no longer interpret $R_{2}$ as the long-term level of mean reversion. Remark that $\pi(0)=0$ if and only if $R_{1} R_{2}>\nu^{2}$.

4. For $R_{0}, R_{1}>0$, the steady-state distribution has a gamma tail on the right and an inverse gamma tail on the left. As a consequence, $\pi$ has finite moments of any order and $\pi(0)=0$. In particular, the first moment equals (see e.g., Jorgensen (1982))

$$
\frac{\sqrt{R_{0} R_{2}}}{\sqrt{R_{1}}} \frac{K_{\xi+1}\left(4 \sqrt{R_{0} R_{1} R_{2}} \nu^{-2}\right)}{K_{\xi}\left(4 \sqrt{R_{0} R_{1} R_{2}} \nu^{-2}\right)}
$$

where $K_{\xi}$ denotes the modified Bessel function of the second kind 9 In general, the first moment of the steady-state distribution will not be exactly equal to $R_{2}$, so we can not interpret $R_{2}$ as the long-term level of mean-reversion. However, the difference is small for standard parameterizations.

\section{Moment explosions}

In this section, we build on the general findings of Lions and Musiela (2007) to investigate moment explosions of the stock price in our model. In order to use (1)-(2) for option pricing, we need $S_{t}$ to be a $\mathbb{Q}$-martingale. The following proposition derives a necessary and sufficient condition.

Proposition 4.1. $S_{t}$ is a $\mathbb{Q}$-martingale if and only if $R_{1} \geq \rho \nu$.

If $R_{1}=0$, then $S_{t}$ is a $\mathbb{Q}$-martingale if and only if $\rho \leq 0$, which is a well known problem with this type of model. While equity markets generally feature a negative correlation between stock returns and volatility, other applications might require a positive correlation. Proposition 4.1 shows that our model can accommodate a positive correlation if $R_{1}$ is sufficiently large. In

\footnotetext{
${ }^{9}$ In Appendix B.1 we provide a tight lower bound (based on Jensen's inequality) for the first moment of the steady-state distribution that does not involve any special functions.
} 
particular, if $R_{1} \geq \nu$, then $S_{t}$ is always a $\mathbb{Q}$-martingale in our model, regardless of $\rho$. Intuitively, the quadratic drift term has a stabilizing effect on the volatility because the speed of mean reversion becomes very large at high volatility levels.

Andersen and Piterbarg (2007) highlight the importance for $S_{t}$ to have finite moments greater than one for pricing contracts with super-linear payoff, which occur frequently in interest rate derivatives markets. Examples include CMS swaps, in-arrears swaps, and Eurodollar futures. Moreover, when using Monte-Carlo simulations to find the price of a derivative, the payoff needs to have finite second order moment in order to derive confidence intervals on the Monte-Carlo estimator with the central limit theorem. The following proposition derives a lower bound on $R_{1}$ such that $S_{t}$ has finite moments of a given order.

Proposition 4.2. Let $m \in \mathbb{R} \backslash[0,1]$.

1. If $R_{1}>\nu\left(\rho m+\sqrt{m^{2}-m}\right)$, then

$$
\mathbb{E}_{t}\left[S_{T}^{m}\right]<\infty, \quad \forall T>t .
$$

If $R_{0} \geq R_{1} R_{2}$, then the statement is also true for $R_{1}=\nu\left(\rho m+\sqrt{m^{2}-m}\right)$.

2. If $R_{1}<\nu\left(\rho m+\sqrt{m^{2}-m}\right)$, then

$$
\mathbb{E}_{t}\left[S_{T}^{m}\right]=\infty, \quad \forall T>t
$$

In particular, if $R_{1}=0$ and $m>1$, then $\mathbb{E}_{t}\left[S_{T}^{m}\right]$ is finite if and only if $\rho \leq-\sqrt{\frac{m-1}{m}}$. A negative correlation has a dampening effect on the moments of the return process, however it must be sufficiently negative in this case for higher moments to exist. For instance, already for $m=2$ we require $\rho \leq-70.71 \%$ in case $R_{1}=0$, which can be quite restrictive. Proposition 4.2 shows that the quadratic term in the drift of $\sigma_{t}$ can take over the role of the negative correlation to stabilize the moments of the return process, which allows the correlation to remain a free parameter. Remark also that for $R_{1}=0$ and $m<0$, we have $\mathbb{E}_{t}\left[S_{T}^{m}\right]=\infty$, regardless of $\rho$.

The seminal work of Lee (2004) relates moment explosions to the asymptotic behaviour of the Black-Scholes implied volatility smile as a function of log-moneyness. Specifically, define the critical moments

$$
m_{+}(T)=\sup \left\{m: S_{T}^{m}<\infty\right\}, \quad m_{-}(T)=\inf \left\{m: S_{T}^{m}<\infty\right\} .
$$

Remark that Proposition 4.2 implies in particular that the critical moments in our model do not depend on the time horizon $T$, so henceforth we omit the time argument and simply write $m_{ \pm}$. Let $\sigma_{B S}(T, x)$ denote the Black-Scholes implied volatility of a European call option with time-to-maturity $T$ and strike price $S_{0} \mathrm{e}^{x}$. Using the formulation of Keller-Ressel (2011), the critical moment formula of Lee (2004) states

$$
\limsup _{x \rightarrow-\infty} \frac{\sigma_{B S}^{2}(T, x)}{|x|}=\frac{\beta\left(-m_{-}\right)}{T} \text { and } \limsup _{x \rightarrow \infty} \frac{\sigma_{B S}^{2}(T, x)}{|x|}=\frac{\beta\left(m_{+}-1\right)}{T},
$$

where we define the decreasing function $\beta: \mathbb{R}_{+} \rightarrow[0,2], x \mapsto 2-4\left(\sqrt{x^{2}+x}-x\right)$. The critical moments in our model can directly be computed using the result of Proposition 4.2. as shown in the following corollary. Note that Black-Scholes implied volatility only makes sense if $S_{t}$ is a $\mathbb{Q}$-martingale, so we only consider the case $R_{1} \geq \rho \nu$, cf. Proposition 4.1 . 
Corollary 4.3. Suppose $S_{t}$ is a $\mathbb{Q}$-martingale, i.e., $R_{1} \geq \rho \nu$.

1. If $|\rho|<1$, then

$$
m_{ \pm}=\frac{1-2 \frac{R_{1}}{\nu} \rho \pm \sqrt{\left(1-2 \frac{R_{1}}{\nu} \rho\right)^{2}+4\left(1-\rho^{2}\right) \frac{R_{1}^{2}}{\nu^{2}}}}{2\left(1-\rho^{2}\right)} .
$$

2. If $\rho=1$, then $m_{-}=-\infty$ and $m_{+}=\frac{R_{1}^{2}}{2 R_{1} \nu-\nu^{2}}$.

3. If $\rho=-1$, then $m_{+}=\infty$ and $m_{-}=\frac{R_{1}^{2}}{-2 R_{1} \nu-\nu^{2}}$.

The critical moment formula (44) and Corollary 4.3 give us important information about the tail behaviour of $x \mapsto \sigma_{B S}^{2}(T, x) T$ in our model. If $|\rho|<1$, then the critical moments are finite, which implies asymptotically linear behaviour of $\sigma_{B S}^{2}(T, x)$ in $x$ for all $T>0$. The slope of the small and large strike tail is controlled by both $\rho$ and $\frac{R_{1}}{\nu}$. For $R_{1}=0$, we get in particular $m_{+}=\left(1-\rho^{2}\right)^{-1}$ and $m_{-}=0$. In this case, $\rho$ only controls the slope of large strike tail, while the slope of the small strike tail is always equal to $\beta(0)=2$. With $R_{1}$ as a free parameter, we can therefore more accurately capture both the small and the large strike tail of the Black-Scholes implied volatility skew.

Remark 4.4. As noted by Lee (2004), the critical moment formula (4) can be useful to facilitate model calibration. Suppose we observe a Black-Scholes implied volatility skew for a range of strikes and a certain maturity $T>0$. From the smallest and largest strike, we can approximately infer $m_{-}$and $m_{+}$, respectively 10 The parameters $\rho$ and $\frac{R_{1}}{\nu}$ can then be calibrated to these implied critical moments using (5). This approach should be seen as a way to get good initial guesses for $\rho$ and $\frac{R_{1}}{\nu}$.

We end this section with an additional result on the two extreme correlation cases.

\section{Proposition 4.5.}

1. If $\rho=-1$ and $R_{0} \geq R_{1} R_{2}$, then

$$
S_{T} \leq S_{t} \exp \left\{\frac{\sigma_{t}}{\nu}+\frac{R_{0} R_{2}}{\nu}(T-t)\right\}, \quad \forall T>t .
$$

2. If $\rho=1, R_{0} \geq R_{1} R_{2}$, and $2 R_{1} \geq \nu$, then

$$
S_{T} \geq S_{t} \exp \left\{-\frac{\sigma_{t}}{\nu}-\frac{R_{0} R_{2}}{\nu}(T-t)\right\}, \quad \forall T>t .
$$

For $\rho=-1$, we know from Proposition 4.1 that $S_{t}$ is a $\mathbb{Q}$-martingale and from Proposition 4.2 that $\mathbb{E}_{t}\left[S_{T}^{m}\right]<\infty$ for all $m>1$ and all $T>t$. If moreover $R_{0} \geq R_{1} R_{2}$, then Proposition 4.5 shows that the stock price becomes bounded form above. Remark that this additional condition is trivially satisfied when $R_{1}=0$. For $\rho=1$, the stock price is a $\mathbb{Q}$-martingale if and only if $R_{1} \geq \nu$, see Proposition 4.1. From Proposition 4.2 we have in this case $\mathbb{E}_{t}\left[S_{T}^{m}\right]<\infty$ for all $m<0$ and all $T>t$. If moreover $R_{0} \geq R_{1} R_{2}$, then the stock price has a lower bound strictly larger than zero. Remark that these results are consistent with the critical moments derived in Corollary 4.3 .

\footnotetext{
${ }^{10}$ Remark that in our model, the critical moments do not depend on the time horizon, while the implied critical moments will likely not be exactly equal for different option maturities, in which case we can for example average the implied critical moments across maturities.
} 


\section{A polynomial diffusion in disguise}

In this section we show how our model can be related to the class of polynomial diffusions using a conveniently chosen change of measure.

Define the process $y_{t}$ through the following stochastic differential equation (SDE)

$$
\mathrm{d} y_{t}=-\frac{1}{2} z^{2} \sigma_{t}^{2} \mathrm{~d} t+z \sigma_{t} \mathrm{~d} W_{t}, \quad y_{0}=0,
$$

with $z=\frac{R_{1}}{\nu}$. Fix a time horizon $T>0$ and define the probability measure $\mathbb{Q}^{z}$ through the following Radon-Nikodym derivative

$$
\frac{\mathrm{d} \mathbb{Q}^{z}}{\mathrm{~d} \mathbb{Q}}=\mathrm{e}^{y_{T}}=\mathrm{e}^{-\frac{1}{2} z^{2} \int_{0}^{T} \sigma_{t}^{2} \mathrm{~d} t+z \int_{0}^{T} \sigma_{t} \mathrm{~d} W_{t}} .
$$

Remark $\mathbb{Q}^{z}=\mathbb{Q}$ if $R_{1}=0$. The following proposition shows that the change of measure is well defined.

Proposition 5.1. The process $\mathrm{e}^{y_{t}}$ is a $\mathbb{Q}$-martingale.

Henceforth $\mathbb{E}_{t}^{z}[\cdot]$ denotes the $\mathcal{F}_{t}$-conditional $\mathbb{Q}^{z}$-expectation. By Girsanov's theorem we have that

$$
W_{t}^{z}=W_{t}-z \int_{0}^{t} \sigma_{s} \mathrm{~d} s \text { and } B_{t}^{z}=B_{t}
$$

are independent $\mathbb{Q}^{z}$-Brownian motions. The $\mathbb{Q}^{z}$-dynamics of $\sigma_{t}$ becomes

$$
\mathrm{d} \sigma_{t}=\left(R_{0} R_{2}+\sigma_{t}\left(R_{1} R_{2}-R_{0}\right)\right) \mathrm{d} t+\nu \sigma_{t} \mathrm{~d} W_{t}^{z} .
$$

The quadratic term in the drift of $\sigma_{t}$ vanishes and $\sigma_{t}$ becomes a polynomial diffusion under $\mathbb{Q}^{z}$. Indeed, it has an affine drift and a linear dispersion function, so that its infinitesimal generator maps polynomials to polynomials of the same degree or less. This allows us to compute all $\mathbb{Q}^{z}$-moments of $\sigma_{t}$ in closed form, which is informative about the $\mathbb{Q}^{z}$-distribution of $\sigma_{t}$. For derivative pricing purposes (see Section 6 for more details), we are not particularly interested in the $\mathbb{Q}^{z}$-distribution of $\sigma_{t}$. Instead, we are mainly interested in the $\mathbb{Q}$-distribution of $x_{t}$ or, equivalently, in the joint $\mathbb{Q}^{z}$-distribution of $x_{t}$ and $y_{t}$. The process $\left(x_{t}, y_{t}, \sigma_{t}\right)$ is not a polynomial diffusion under $\mathbb{Q}^{z}$, because the drift of $x_{t}$ and $y_{t}$ contains a quadratic term $\sigma_{t}^{2}$ :

$$
\begin{aligned}
\mathrm{d} x_{t} & =\left(z \rho-\frac{1}{2}\right) \sigma_{t}^{2} \mathrm{~d} t+\sigma_{t}\left(\rho \mathrm{d} W_{t}^{z}+\sqrt{1-\rho^{2}} \mathrm{~d} B_{t}^{z}\right), \\
\mathrm{d} y_{t} & =\frac{1}{2} z^{2} \sigma_{t}^{2} \mathrm{~d} t+z \sigma_{t} \mathrm{~d} W_{t}^{z}, \\
\mathrm{~d} \sigma_{t} & =\left(R_{0} R_{2}+\sigma_{t}\left(R_{1} R_{2}-R_{0}\right)\right) \mathrm{d} t+\nu \sigma_{t} \mathrm{~d} W_{t}^{z} .
\end{aligned}
$$

However, by augmenting the state with $\sigma_{t}^{2}$, we can see that $\left(x_{t}, y_{t}, \sigma_{t}, \sigma_{t}^{2}\right)$ jointly becomes a polynomial diffusion under $\mathbb{Q}^{z}$ since $\sigma_{t}^{2}$ has the following dynamics

$$
\mathrm{d} \sigma_{t}^{2}=\left(2 R_{0} R_{2} \sigma_{t}+\sigma_{t}^{2}\left(2 R_{1} R_{2}-2 R_{0}+\nu^{2}\right)\right) \mathrm{d} t+2 \nu \sigma_{t}^{2} \mathrm{~d} W_{t}^{z} .
$$

This observation makes it possible to calculate all conditional $\mathbb{Q}^{z}$-moments of $\left(x_{t}, y_{t}, \sigma_{t}\right)$ in closed form. Before we do this, we first introduce some notation. Denote for $m, n \in \mathbb{N}$ by $\operatorname{Pol}_{m}\left(\mathbb{R}^{n}\right)$ the 
space of polynomials on $\mathbb{R}^{n}$ with total degree at most $m$. Define the subspace $P_{m} \subset \operatorname{Pol}_{2 \mathrm{~m}}\left(\mathbb{R}^{3}\right)$ of trivariate polynomials as

$$
P_{m}=\left\{(x, y, z) \mapsto p(x, y) q(z) \mid p \in \operatorname{Pol}_{m}\left(\mathbb{R}^{2}\right), q \in \operatorname{Pol}_{2(m-\operatorname{deg}(p))}(\mathbb{R})\right\},
$$

where $\operatorname{deg}(\cdot)$ denotes the total degree of a polynomial. The following lemma provides the dimension of $P_{m}$, i.e. the number of linearly independent polynomials in $P_{m}$.

Lemma 5.2. The dimension of $P_{m}$ is

$$
d_{m}=\operatorname{dim}\left(P_{m}\right)=\frac{1}{3} m^{3}+\frac{3}{2} m^{2}+\frac{13}{6} m+1 .
$$

The following proposition provides an explicit formula for the conditional $\mathbb{Q}^{z}$-moments of $\left(x_{t}, y_{t}, \sigma_{t}\right)$, which will be the cornerstone of the derivative pricing approximation method in Section 6 .

Proposition 5.3. The infinitesimal generator $\mathcal{G}$ of the process $\left(x_{t}, y_{t}, \sigma_{t}\right)$ under $\mathbb{Q}^{z}$ leaves $P_{m}$ invariant. That is, there exists a matrix $G_{m} \in \mathbb{R}^{d_{m} \times d_{m}}$, such that $\mathcal{G} H_{m}=G_{m} H_{m}$, where $H_{m}=\left(h_{1}, \ldots, h_{d_{m}}\right)^{\top}$ denotes a vector of polynomial basis functions for $P_{m}$. As a consequence, we have for any $t \leq T$

$$
\mathbb{E}_{t}^{z}\left[H_{m}\left(x_{T}, y_{T}, \sigma_{T}\right)\right]=\mathrm{e}^{G_{m}(T-t)} H_{m}\left(x_{t}, y_{t}, \sigma_{t}\right) .
$$

The matrix $G_{m}$ is straightforward to construct in practice by choosing $H_{m}$ to be a monomial basis and then collecting terms according to their exponents in the vector of polynomials $\mathcal{G} H_{m}$, see equation (22) in the Appendix.

\section{Derivative pricing}

In this section we show how European style derivatives on the stock price $\mathrm{e}^{x_{T}}$ can efficiently be computed using the available $\mathbb{Q}^{z}$-moments of $\left(x_{T}, y_{T}\right)$.

\subsection{Polynomial payoff approximation}

Consider a derivative on the stock price with payoff $F\left(\mathrm{e}^{x_{T}}\right)$ at time $T>0$, for some integrable payoff function $F$. The price at time 0 is given by

$$
\pi=\mathbb{E}_{0}\left[F\left(\mathrm{e}^{x_{T}}\right)\right]=\mathbb{E}_{0}^{z}\left[\mathrm{e}^{-y_{T}} F\left(\mathrm{e}^{x_{T}}\right)\right] .
$$

The auxiliary process $y_{t}$ can therefore be interpreted as a stochastic discount rate under the new measure. The positive correlation between $y_{t}$ and $\sigma_{t}$ provides a dampening effect on the 'discounted' payoff under the new measure, which is the equivalent of the dampening effect of the quadratic drift term of $\sigma_{t}$ that disappeared with the measure change.

The conditional $\mathbb{Q}$-distribution of $x_{T}$ is not known, but we do know all the conditional $\mathbb{Q}^{z}$ moments of $\left(x_{T}, y_{T}\right)$ thanks to the moment formula (9). Therefore, we can approximate the derivative price by approximating the function $(x, y) \mapsto e^{-y} F\left(\mathrm{e}^{x}\right)$ with a polynomial $p_{n} \in$ $\operatorname{Pol}_{n}\left(\mathbb{R}^{2}\right)$, for some $n \in \mathbb{N}$. We would like the polynomial approximation to be most accurate for the values that $\left(x_{T}, y_{T}\right)$ is most likely to take under $\mathbb{Q}^{z}$, since they contribute the most to the 
right hand side of (10). This motivates the following least-squares approach to determine the approximating polynomial

$$
p_{n}=\underset{p \in \operatorname{Pol}_{n}\left(\mathbb{R}^{2}\right)}{\arg \min } \int_{\mathbb{R}^{2}}\left(e^{-y} F\left(e^{x}\right)-p(x, y)\right)^{2} w(x, y) \mathrm{d} x \mathrm{~d} y,
$$

where $w$ is an auxiliary probability density function which proxies the unknown $\mathbb{Q}^{z}$-density of $\left(x_{T}, y_{T}\right) 11$ Put differently, $p_{n}$ is the orthogonal projection of $(x, y) \mapsto e^{-y} F\left(\mathrm{e}^{x}\right)$ on the space of bivariate polynomials of total degree $n$ or less in a weighted Hilbert function space with weight $w$. If we denote by $\vec{p}_{n} \in \mathbb{R}^{d_{n}}$ the vector representation of $p_{n}$ with respect to the basis $H_{n}$, the option price approximation becomes

$$
\pi \approx \pi_{n}=\vec{p}_{n}^{\top} \mathrm{e}^{G_{n} T} H_{n}\left(x_{0}, y_{0}, \sigma_{0}\right) .
$$

In Section 6.2 we show how to choose $w$ and in Section 6.3 we solve the optimization problem in (11).

\subsection{The auxiliary density}

It remains to choose a good auxiliary density $w$. We use an approach that closely resembles the Gaussian mixture specification of Ackerer and Filipovic (2019). Conditional on the trajectory $\left\{W_{t}^{z}, t \leq T\right\}$, the $\mathbb{Q}^{z}$-density function of the random variable $\left(x_{T}, y_{T}\right)$ can be formally written as

$$
(x, y) \mapsto \phi_{M_{T}, V_{T}}(x) \delta\left(y-y_{T}\right),
$$

where $\phi_{M_{T}, V_{T}}$ denotes the density function of a Gaussian distribution with mean $M_{T}$ and variance $V_{T}, \delta$ denotes the Dirac delta function, and

$$
M_{T}=x_{0}+\left(-\frac{1}{2}+z \rho\right) \int_{0}^{T} \sigma_{s}^{2} \mathrm{~d} s+\rho \int_{0}^{T} \sigma_{s} \mathrm{~d} W_{s}^{z}, \quad V_{T}=\left(1-\rho^{2}\right) \int_{0}^{T} \sigma_{s}^{2} \mathrm{~d} s
$$

The true $\mathbb{Q}^{z}$-density function of $\left(x_{T}, y_{T}\right)$ can therefore be expressed as

$$
(x, y) \mapsto \mathbb{E}_{0}^{z}\left[\phi_{M_{T}, V_{T}}(x) \delta\left(y-y_{T}\right)\right] .
$$

We specify the auxiliary density as

$$
w(x, y)=\sum_{k=1}^{\mathcal{K}} w^{(k)} \phi_{m^{(k)}, v^{(k)}}(x) \delta\left(y-y^{(k)}\right),
$$

where $m^{(k)}, y^{(k)} \in \mathbb{R}, v^{(k)} \in \mathbb{R}_{+}, w^{(k)} \in[0,1], k=1, \ldots, \mathcal{K}$, are constants to be determined subject to $\sum_{k=1}^{\mathcal{K}} w^{(k)}=1$. The quadruplets $\left(w^{(k)}, m^{(k)}, v^{(k)}, y^{(k)}\right)$ represent a discretization of the $\mathbb{Q}^{z}$-distribution of $\left(M_{T}, V_{T}, y_{T}\right)$ in $\mathcal{K} \geq 1$ mass points, which can be obtained by discretizing the single source of uncertainty $\left\{W_{t}^{z}, t \leq T\right\}$. Specifically, we use the IJK scheme of Kahl and Jäckel (2006) with $d \geq 1$ equidistant time steps to obtain the following discretization scheme for $\sigma_{t}$,

\footnotetext{
${ }^{11}$ We assume that $w$ is such that the double integral in (11) is finite for all $p \in \operatorname{Pol}_{n}\left(\mathbb{R}^{2}\right)$.
} 
$M_{t}, V_{t}$, and $y_{t}$ :

$$
\begin{aligned}
& \hat{\sigma}_{n+1}=\hat{\sigma}_{n}+\left(R_{0} R_{2}+\hat{\sigma}_{n}\left(R_{1} R_{2}-R_{0}\right)\right) \Delta+\nu \hat{\sigma}_{n} \sqrt{\Delta} Z_{n+1}+\frac{1}{2} \nu^{2} \hat{\sigma}_{n}\left(\Delta Z_{n+1}^{2}-\Delta\right), \\
& \hat{M}_{n+1}=\hat{M}_{n+1}+\left(-\frac{1}{2}+z \rho\right) \frac{\hat{\sigma}_{n+1}^{2}+\hat{\sigma}_{n}^{2}}{2} \Delta+\rho \hat{\sigma}_{t_{n}} \sqrt{\Delta} Z_{n+1}, \\
& \hat{V}_{n+1}=\hat{V}_{n}+\left(1-\rho^{2}\right) \frac{\hat{\sigma}_{n+1}^{2}+\hat{\sigma}_{n}^{2}}{2} \Delta, \\
& \hat{y}_{n+1}=\hat{y}_{n}+\frac{1}{2} z^{2} \frac{\hat{\sigma}_{n+1}^{2}+\hat{\sigma}_{n}^{2}}{2} \Delta+z \hat{\sigma}_{n} \sqrt{\Delta} Z_{n+1},
\end{aligned}
$$

where $\Delta=\frac{T}{d}$ is the step size, $\left(Z_{1}, \ldots, Z_{d}\right)$ is a $d$-dimensional standard normal random variable, and $\hat{\sigma}_{0}=\sigma_{0}, \hat{M}_{0}=M_{0}, \hat{V}_{0}=V_{0}, \hat{y}_{0}=y_{0}$. If we are given $\mathcal{K}$ weighted samples $\left(w^{(k)}, Z_{1}^{(k)}, \ldots, Z_{d}^{(k)}\right), k=1 \ldots, \mathcal{K}$, of the random variable $\left(Z_{1}, \ldots, Z_{d}\right)$, then by plugging them into the above scheme we obtain the quadruplets $\left(w^{(k)}, m^{(k)}, v^{(k)}, y^{(k)}\right)$. As highlighted by Ackerer and Filipović (2019), raw Monte-Carlo simulation with $w^{(k)} \equiv 1 / \mathcal{K}$ requires far too many samples to produce an accurate approximation of the distribution. Instead, deterministic discretizations of the $d$-dimensional standard normal distribution, such as the quantization techniques of Pagès and Printems (2003) or Gaussian cubature rules, are preferred in order to keep $\mathcal{K}$ small. In the numerical study in Section 7, we use the multivariate Gauss-Hermite quadrature method described in Jäckel (2005) to obtain the weighted samples $\left(w^{(k)}, Z_{1}^{(k)}, \ldots, Z_{d}^{(k)}\right)$. The advantage of Gauss-Hermite quadrature is that the tails of the distribution are accurately captured, which is important for the stability of our approximation method as $n$, the total polynomial degree of the approximation, increases.

\subsection{The optimal polynomial}

Now that we have specified the auxiliary density function, we can solve the optimization problem in (11). Denote by $B_{n}=\left(b_{1}, \ldots, b_{N_{n}}\right)^{\top}, N_{n}=\left(\begin{array}{c}n+2 \\ 2\end{array}\right)$, a vector of polynomial basis functions for $\operatorname{Pol}_{n}\left(\mathbb{R}^{2}\right)$. We can rewrite (11) as

$$
c_{n}=\underset{c \in \mathbb{R}^{N_{n}}}{\arg \min } \int_{\mathbb{R}^{2}}\left(e^{-y} F\left(e^{x}\right)-c^{\top} B_{n}(x, y)\right)^{2} w(x, y) \mathrm{d} x \mathrm{~d} y .
$$

Proposition 6.1. The unique solution of (14) is $c_{n}=D^{-1} f$, with

$$
D_{i, j}=\int_{\mathbb{R}^{2}} b_{i}(x, y) b_{j}(x, y) w(x, y) \mathrm{d} x \mathrm{~d} y, \quad f_{i}=\int_{\mathbb{R}^{2}} e^{-y} F\left(\mathrm{e}^{x}\right) b_{i}(x, y) w(x, y) \mathrm{d} x \mathrm{~d} y,
$$

for $i, j=1, \ldots, N_{n}$.

Without loss of generality, we assume that $B_{n}$ is a monomial basis with $b_{i}(x, y)=x^{\alpha_{i}} y^{\beta_{i}}$, for exponents $\alpha_{i}, \beta_{i} \in \mathbb{N}$ such that $\alpha_{i}+\beta_{i} \leq n, i=1, \ldots, N_{n}$. Plugging (13) in the expression for $D_{i, j}$ in (15) gives

$$
D_{i, j}=\sum_{k=1}^{K} w^{(k)}\left(y^{(k)}\right)^{\beta_{i}+\beta_{j}} \int_{\mathbb{R}} x^{\alpha_{i}+\alpha_{j}} \phi_{m^{(k)}, v^{(k)}}(x) \mathrm{d} x .
$$


The remaining integral is simply the $\left(\alpha_{i}+\alpha_{j}\right)$-th moment of the univivariate Gaussian distribution, which is known in closed form 12 The elements of the vector $f$ become

$$
f_{i}=\sum_{k=1}^{K} w^{(k)} \mathrm{e}^{-y^{(k)}}\left(y^{(k)}\right)^{\beta_{i}} \int_{\mathbb{R}} F\left(\mathrm{e}^{x}\right) x^{\alpha_{i}} \phi_{m^{(k)}, v^{(k)}}(x) \mathrm{d} x .
$$

In general, the integral in (16) has to be computed numerically, for example using GaussHermite quadrature. For specific payoff functions, the integral can be computed in closed form. For example, the following proposition derives a recursive formula for the case of a European call option.

Proposition 6.2. Suppose $F(x)=\left(\mathrm{e}^{x}-K\right)^{+}$, for some $K>0$. The integral

$$
I_{n}^{(k)}=\int_{\mathbb{R}} F\left(\mathrm{e}^{x}\right) x^{n} \phi_{m^{(k)}, v^{(k)}}(x) \mathrm{d} x,
$$

satisfies the following recursion for $n \geq 1$

$$
\begin{aligned}
& I_{n}^{(k)}=\left(m^{(k)}+v^{(k)}\right) I_{n-1}^{(k)}+v^{(k)}(n-1) I_{n-2}^{(k)}+K v^{(k)} J_{n-1}^{(k)}, \\
& J_{n}^{(k)}=m^{(k)} J_{n-1}^{(k)}+v^{(k)}(n-1) J_{n-2}^{(k)}+\sqrt{v^{(k)}}(\log (K))^{n-1} \phi\left(\xi^{(k)}\right),
\end{aligned}
$$

with $\xi^{(k)}=\frac{m^{(k)}-\log (K)}{\sqrt{v^{(k)}}}$, $\phi$ the standard normal density, and starting values

$$
I_{-1}^{(k)}=0, \quad I_{0}^{(k)}=\mathrm{e}^{m^{(k)}+\frac{1}{2} v^{(k)}} \Phi\left(\xi^{(k)}+\sqrt{v^{(k)}}\right)-K \Phi\left(\xi^{(k)}\right), \quad J_{-1}^{(k)}=0, \quad J_{0}^{(k)}=\Phi\left(\xi^{(k)}\right),
$$

with $\Phi$ the standard normal cumulative distribution function.

\section{Numerical study}

In this section we investigate the numerical accuracy of the option price approximation proposed in the previous section.

We set the model parameters as $R_{0}=R_{1}=5, \nu=1, R_{2}=\sigma_{0}=0.20, \rho=-0.5, x_{0}=0$. These are realistic parameters that produce a volatility process with strong mean-reversion and a high volatility of volatility that can cause occasional spikes, see for example Figure 2 for a simulated (under $\mathbb{Q}$ ) trajectory. Consider a European call option with time-to-maturity $T \in\{1 / 12,2 / 12\}$ and $\log$-strike $\log (K) \in\{-0.1,0,0.1\}$. Figure 3a and 3b plot the option price approximations $\pi_{n}$ for $n$ ranging from 1 to 10 . We set $d=1$, and use the Gauss-Hermite quadrature rule to obtain a discretization of the univariate standard normal distribution in $\mathcal{K}=15$ points. As a benchmark, we also run a Monte-Carlo simulation with $10^{6}$ sample paths 13 For all strikes and maturities considered, $\pi_{n}$ converges to within the confidence bands of the Monte-Carlo estimator with $n \leq 10$. For $n<3$, the pricing error is most noticeable for the low strike option (i.e., the in-the-money call). This is not surprising, since the true log-return distribution is negatively

\footnotetext{
${ }^{12}$ In Appendix B.2 we provide a simple formula for the moments of the Gaussian distribution.

${ }^{13}$ We use a quadratic polynomial approximation of the discounted payoff as a control variate to substantially reduce the variance of the Monte-Carlo estimator. To determine the polynomial approximation, we perform a linear regression with the simulated trajectories. This is similar to the polynomial approximation in Section 6 , where we now use the simulated empirical distribution as auxiliary distribution.
} 


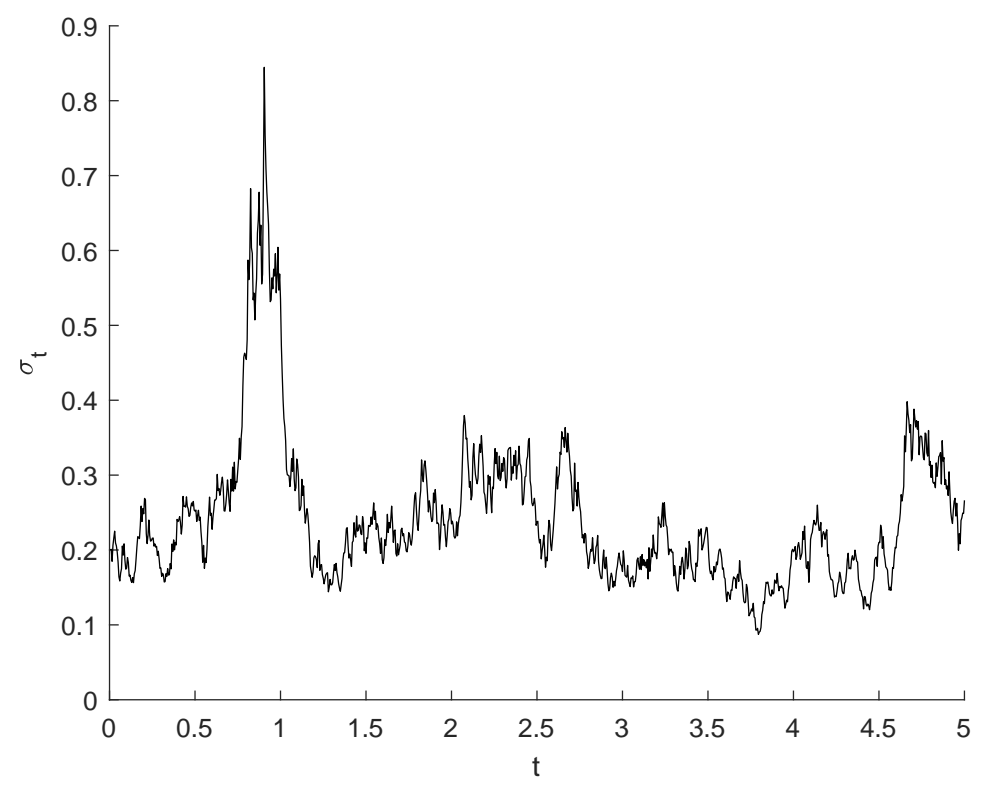

Figure 2: Simulated trajectory for $\sigma_{t}$ with parameters $R_{0}=R_{1}=5, R_{2}=\sigma_{0}=0.20, \nu=1$.

skewed $(\rho<0)$. Therefore, approximations which do not take into account at least third order moments will be far off for low strike options. The results are robust to changes in the number of discretization points $\mathcal{K}$, as long as it is not too small. If $\mathcal{K}$ is chosen very small (say, $\mathcal{K}=3$ ), then the approximation blows up for larger $n$. Intuitively, for small $\mathcal{K}$ the auxiliary distribution $w$ has very thin tails and the polynomial approximation of the discounted payoff will therefore only be accurate over a small domain. Since the true probability distribution assigns considerable weight outside of this domain, the polynomial approximation will blow up quickly. In Figure $3 \mathrm{c}$ and $3 \mathrm{~d}$, we do the same exercise with $d=2$. For the auxiliary distribution, we use the Gauss-Hermite quadrature rule with 15 points in each dimension, which gives a total of $\mathcal{K}=15^{2}=225$ points 14 Compared to the case $d=1$, the approximations converges faster to the true price. However, this comes at a computational cost because the number of discretization points in the auxiliary distribution is much larger.

\section{Conclusion}

We have introduced a new stochastic volatility model featuring a volatility process with a quadratic drift and a linear dispersion function. We have shown that the quadratic term in the drift is important to control moment explosions in the stock price and, in particular, the small strike tail of the Black-Scholes implied volatility skew. The volatility process has a stationary distribution that belongs to the class of Generalized Inverse Gaussian distributions, which arises frequently in the empirical literature on volatility modeling. In order to make the model tractable, we introduced a change of measure such that the model fits into the class of polynomial diffusions, which opened the door to polynomial expansion methods to accurately approximate option prices.

\footnotetext{
${ }^{14}$ Using the pruning method described in Jäckel (2005), we can reduce the number of discretization points to 185 by omitting the 'corner' points that carry a very small weight.
} 


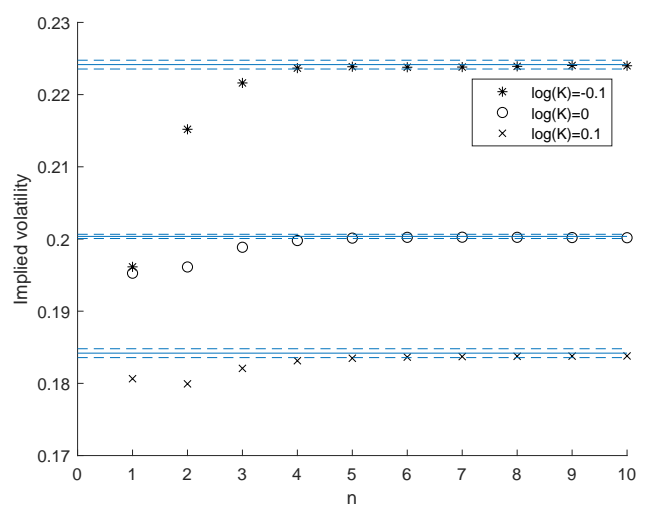

(a) $T=1 / 12, d=1$

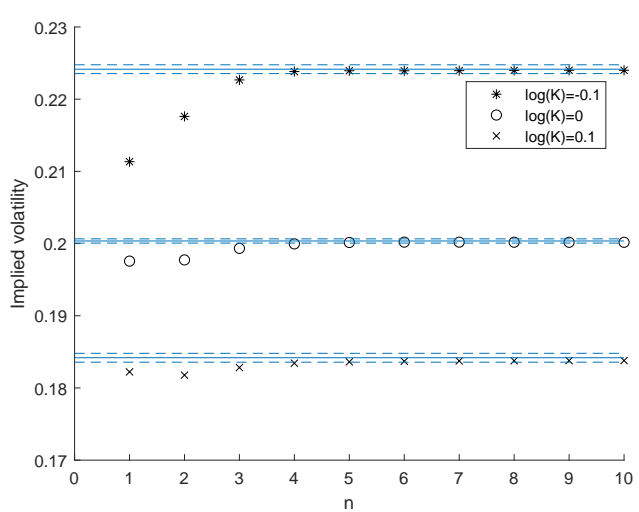

(c) $T=1 / 12, d=2$

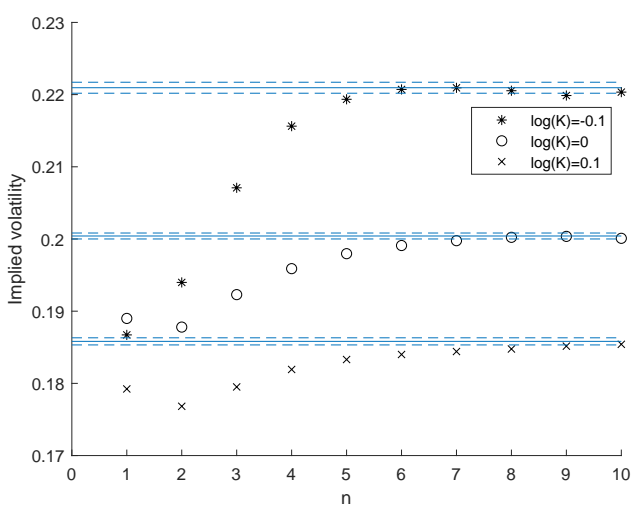

(b) $T=2 / 12, d=1$

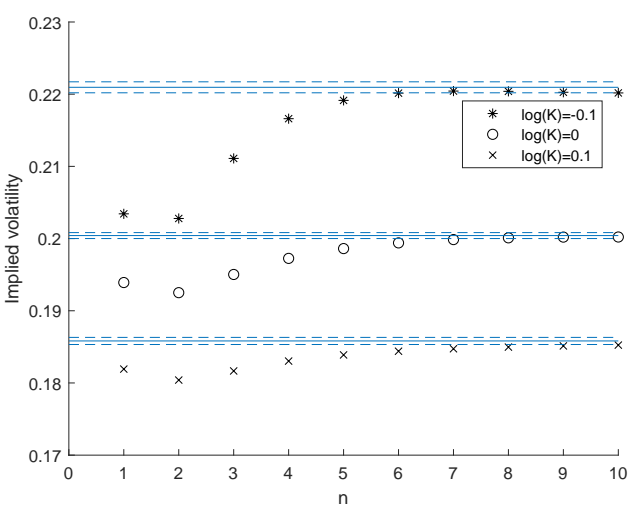

(d) $T=2 / 12, d=2$

Figure 3: Black-Scholes implied volatilities of approximated European call option prices with time-to-maturity of one and two months for varying number of terms $n$ in the series. Solid blue lines are Monte-Carlo estimators using $10^{6}$ sample paths and the dashed blue lines are the corresponding $99 \%$ confidence intervals. We use a quadratic polynomial approximation of the discounted payoff as a control variate to substantially reduce the variance of the MonteCarlo estimator. The top row uses a single time step discretization to construct the auxiliary distribution, while the bottom row uses two equidistant time steps. 


\section{A Proofs}

\section{A.1 Proof of Proposition 2.1}

We start by showing that (2) has a unique $(0, \infty)$-valued solution. We denote by

$$
\mu(x)=\left(R_{0}+R_{1} x\right)\left(R_{2}-x\right) \quad \text { and } \quad \Sigma(x)=\nu x
$$

the drift and dispersion function of $\sigma_{t}$, respectively. Since $\mu$ and $\Sigma$ are polynomials, they are in particular locally Lipschitz continuous. Hence, strong uniqueness holds for solutions of (2), see e.g. (Karatzas and Shreve, 1991, Theorem 2.5). The dispersion function satisfies a linear growth condition

$$
|\Sigma(x)|^{2} \leq K_{1}\left(1+|x|^{2}\right),
$$

for $K_{1} \geq \nu$. The drift function does not satisfy a linear growth condition, so the classical existence result of Itô (see e.g., (Karatzas and Shreve, 1991, Theorem 2.9)) does not apply. However, since the quadratic term in $\mu$ has a negative coefficient, $\mu$ does satisfy

$$
x \mu(x) \leq K_{2}\left(1+|x|^{2}\right),
$$

for some $K_{2} \geq 0$. Hence, there exists a unique global solution to (2), cf. (Kloeden and Platen, 1995, Chapter 4.5, p.135). It remains to verify that the solution stays in $(0, \infty)$, which we proof using a comparison theorem. Consider the logistic diffusion

$$
\mathrm{d} X_{t}=\left(-R_{1} X_{t}^{2}+\left(R_{1} R_{2}-R_{0}\right) X_{t}\right) \mathrm{d} t+\nu X_{t} \mathrm{~d} W_{t}, \quad X_{0}=\sigma_{0} .
$$

This SDE has a unique solution given by

$$
X_{t}=\frac{Y_{t}}{1+R_{1} \int_{0}^{t} Y_{s} \mathrm{~d} s}, \quad \text { with } \quad Y_{t}=X_{0} \mathrm{e}^{\left(R_{1} R_{2}-R_{0}-\frac{1}{2} \nu^{2}\right) t+\nu W_{t}} .
$$

Notice that, since $R_{1} \geq 0$, we have $X_{t}>0$ for all $t \geq 0$. Using a comparison theorem (Ikeda and Watanabe, 1989, Chapter VI, Theorem 1.1) and $R_{0} R_{2} \geq 0$ gives a.s. $\sigma_{t} \geq X_{t}>0$ for all $t \geq 0$.

Remark A.1. Notice that, if $R_{1}<0$, then $X_{t}$ explodes in finite time and therefore $\sigma_{t}$ as well. The assumption $R_{1} \geq 0$ is therefore crucial to guarantee existence of a global solution.

Next, we show that the stochastic integrals in (1) are well defined by showing that

$$
\mathbb{E}_{t}\left[\int_{t}^{T} \sigma_{s}^{2} \mathrm{~d} s\right]<\infty, \quad \forall T \geq t
$$

To this end, consider the SDE

$$
\mathrm{d} Z_{t}=\left(R_{0} R_{2}+\left(R_{1} R_{2}-R_{0}\right) Z_{t}\right) \mathrm{d} t+\nu Z_{t} \mathrm{~d} W_{t}, \quad Z_{0}=\sigma_{0},
$$

which has $Z_{t}=Y_{t}\left(1+R_{0} R_{2} \int_{0}^{t} Y_{s}^{-1} \mathrm{~d} s\right)$ as unique solution. Using a comparison theorem (Ikeda and Watanabe, 1989, Chapter VI, Theorem 1.1) and $R_{1} \geq 0$ gives a.s. $\sigma_{t} \leq Z_{t}$ for all $t \geq 0$. Since $Z_{t}$ is a polynomial diffusion, it has finite moments of any order. Therefore we have

$$
\mathbb{E}_{t}\left[\int_{t}^{T} \sigma_{s}^{2} \mathrm{~d} s\right] \leq \int_{t}^{T} \mathbb{E}_{t}\left[Z_{s}^{2}\right] \mathrm{d} s<\infty, \quad \forall T \geq t .
$$

From the comparison arguments used in first and second part of this proof, we also obtain the following pathwise bounds on $\sigma_{t}$

$$
X_{t} \leq \sigma_{t} \leq Z_{t}, \quad \text { a.s. }
$$




\section{A.2 Proof of Proposition 3.1}

Using the Fokker-Planck equation we have that $\pi$ must satisfy the following second order linear ODE

$$
\frac{\nu^{2}}{2} \frac{\mathrm{d}^{2}}{\mathrm{~d} x^{2}}\left[x^{2} \pi(x)\right]=\frac{\mathrm{d}}{\mathrm{d} x}\left[\left(R_{0}+R_{1} x\right)\left(R_{2}-x\right) \pi(x)\right] .
$$

Making the Ansatz $\pi=x^{\gamma} \exp \left\{\alpha \frac{1}{x}+\beta x\right\}$, for some constants $\alpha, \beta$, and $\gamma$, and collecting terms gives

$$
\pi(x)=C x^{\xi-1} \exp \left\{-2 \frac{R_{0} R_{2}}{\nu^{2}} \frac{1}{x}-2 \frac{R_{1}}{\nu^{2}} x\right\},
$$

where $C$ is a constant to be determined such that $\pi$ integrates to one.

Remark A.2. The motivation for this Ansatz comes from the special cases $R_{1}=0$ and $R_{0}=0$. If $R_{1}=0$, then $\sigma_{t}$ is a GARCH diffusion, which is known to have the inverse gamma distribution as steady-state distribution. If $R_{0}=0$, then $\sigma_{t}$ is a logistic diffusion, which is known to have the gamma distribution as steady-state distribution if $2 R_{1} R_{2}>\nu^{2}$. Therefore, the steady-state distribution of $\sigma_{t}$ must contain the gamma and inverse gamma distribution as special cases.

If $R_{0}, R_{1}>0$, then $\pi$ is a generalized inverse Gaussian distribution and the normalization constant becomes (see e.g., Jorgensen (1982))

$$
C=\frac{\left(\frac{R_{1}}{R_{0} R_{2}}\right)^{\xi / 2}}{2 K_{\xi}\left(4 \sqrt{R_{0} R_{1} R_{2}} \nu^{-2}\right)},
$$

where $K_{\xi}$ denotes the modified Bessel function of the second kind. If $R_{0}=0$ and $2 R_{1} R_{2}>\nu^{2}$, then $\pi$ is a gamma density and $C$ therefore becomes

$$
C=\frac{\left(\frac{2 R_{1}}{\nu^{2}}\right)^{\xi}}{\Gamma(\xi)}
$$

where $\Gamma$ denotes the gamma function. Note that the condition $2 R_{1} R_{2}>\nu^{2}$ is equivalent to $\xi>0$.

For $R_{0}=0$ and $2 R_{1} R_{2} \leq \nu^{2}$, recall from (18) that we have almost surely the following upper bound

$$
\sigma_{t} \leq Z_{t}=\sigma_{0} \mathrm{e}^{\left(R_{1} R_{2}-\frac{1}{2} \nu^{2}\right) t+\nu W_{t}} .
$$

If $2 R_{1} R_{2} \geq \nu^{2}$, then $\mathrm{e}^{\left(R_{1} R_{2}-\frac{1}{2} \nu^{2}\right) t+\nu W_{t}} \rightarrow 0$ a.s. for $t \rightarrow \infty$ and therefore we have $\sigma_{t} \rightarrow 0$ a.s. for $t \rightarrow \infty$.

\section{A.3 Proof of Proposition 4.1}

By Theorem 2.4(i) in Lions and Musiela (2007), $S_{t}$ is a $\mathbb{Q}$-martingale if

$$
\lim _{x \rightarrow \infty} \frac{\rho \nu x^{2}+\left(R_{0}+R_{1} x\right)\left(R_{2}-x\right)}{x}<\infty .
$$


The limit can be rewritten as

$$
\begin{aligned}
\lim _{x \rightarrow \infty} \frac{\rho \nu x^{2}+\left(R_{0}+R_{1} x\right)\left(R_{2}-x\right)}{x} & =\lim _{x \rightarrow \infty} \frac{\left(\rho \nu-R_{1}\right) x^{2}+\left(R_{1} R_{2}-R_{0}\right) x+R_{0} R_{2}}{x} \\
& =\lim _{x \rightarrow \infty}\left(\rho \nu-R_{1}\right) x+R_{1} R_{2}-R_{0} .
\end{aligned}
$$

Therefore, $S_{t}$ is a $\mathbb{Q}$-martingale if $R_{1} \geq \rho \nu$. Indeed, if $R_{1}>\rho \nu$ the limit is $-\infty$ and if $R_{1}=\rho \nu$ the limit is $R_{1} R_{2}-R_{0}$.

Conversely, by Theorem 2.4(ii) in Lions and Musiela (2007), $S_{t}$ is not a $\mathbb{Q}$-martingale if

$$
\lim _{x \rightarrow \infty} \frac{\rho \nu x^{2}+\left(R_{0}+R_{1} x\right)\left(R_{2}-x\right)}{\phi(x)}>0,
$$

for some smooth, positive, and increasing function $\phi$ such that $\int_{\epsilon}^{\infty} \frac{1}{\phi(x)} \mathrm{d} x<\infty, \epsilon>0$. Choosing $\phi(x)=x^{2}$ gives

$$
\lim _{x \rightarrow \infty} \frac{\rho \nu x^{2}+\left(R_{0}+R_{1} x\right)\left(R_{2}-x\right)}{x^{2}}=\rho \nu-R_{1} .
$$

Therefore, $S_{t}$ is not a $\mathbb{Q}$-martingale if $R_{1}<\rho \nu$.

\section{A.4 Proof of Proposition 4.2}

1. By Theorem 2.5 in Lions and Musiela (2007), we need to show that there exists an $A \geq 0$ such that 15

$$
\lim _{x \rightarrow \infty}-\frac{1}{2} A^{2} \nu^{2} x^{2}-A\left[m \rho \nu x^{2}+\left(R_{0}+R_{1} x\right)\left(R_{2}-x\right)\right]-\frac{m^{2}-m}{2} x^{2}>-\infty .
$$

The limit can be rewritten as

$$
\begin{aligned}
& \lim _{x \rightarrow \infty}-\frac{1}{2} A^{2} \nu^{2}+x^{2}-A\left[m \rho \nu x^{2}+\left(R_{0}+R_{1} x\right)\left(R_{2}-x\right)\right]-\frac{m^{2}-m}{2} x^{2} \\
= & \lim _{x \rightarrow \infty}\left(-\frac{1}{2} A^{2} \nu^{2}-A\left[m \rho \nu-R_{1}\right]-\frac{m^{2}-m}{2}\right) x^{2}-A\left(R_{1} R_{2}-R_{0}\right) x-A R_{0} R_{2} .
\end{aligned}
$$

Define the parabola $f(u)=-\frac{1}{2} u^{2} \nu^{2}-u\left[m \rho \nu-R_{1}\right]-\frac{m^{2}-m}{2}$. If

$$
m \rho \nu-R_{1}<-\nu \sqrt{m^{2}-m}
$$

then $f$ has two distinct positive roots

$$
A_{ \pm}=\frac{m \rho \nu-R_{1} \pm \sqrt{\left(m \rho \nu-R_{1}\right)^{2}-\nu^{2}\left(m^{2}-m\right)}}{-\nu^{2}} .
$$

From (201) it becomes clear that if we pick $A \in\left(A_{-}, A_{+}\right)$, then (19) is satisfied.

If

$$
m \rho \nu-R_{1}=-\nu \sqrt{m^{2}-m}
$$

\footnotetext{
${ }^{15}$ The paper of Lions and Musiela (2007) contains some typos that are relevant for the derivation of this proof. Specifically, in equation (26), the function $\beta$ should be defined as $\beta(x)=m \rho \mu(x) x+b(x)$ instead of $\beta(x)=$ $m \mu(x) x+b(x)$. In equation (28), the $\mu$ in the last term has to be replaced by $m$.
} 
then $f$ only has a single root

$$
A_{0}=\frac{m \rho \nu-R_{1}}{-\nu^{2}}=\frac{\sqrt{m^{2}-m}}{\nu}>0 .
$$

For all other values, $f$ will be negative. In other words, any value other than $A_{0}$ will make (20) equal to $-\infty$. It remains to check what happens to (20) for $A=A_{0}$

$$
\lim _{x \rightarrow \infty} f\left(A_{0}\right) x^{2}-A_{0}\left(R_{1} R_{2}-R_{0}\right) x-A_{0} R_{0} R_{2}=\lim _{x \rightarrow \infty}-A_{0}\left(R_{1} R_{2}-R_{0}\right) x-A_{0} R_{0} R_{2} .
$$

Therefore, the limit will be larger than $-\infty$ if $R_{0} \geq R_{1} R_{2}$.

2. Follows directly from Theorem 2.6 in Lions and Musiela (2007) and

$$
\lim _{x \rightarrow \infty} \frac{\nu x}{x}=\nu, \quad \lim _{x \rightarrow \infty} \frac{\left(R_{0}+R_{1} x\right)\left(R_{2}-x\right)}{x^{2}}=-R_{1} .
$$

\section{A.5 Proof of Corollary 4.3}

Define the function $f(m)=\rho m+\sqrt{m^{2}-m}$ on $\mathbb{R} \backslash(0,1)$. It is readily verified that $f$ is increasing on $[1, \infty)$ with $f(1)=\rho$ and decreasing on $(-\infty, 0]$ with $f(0)=0$.

If $|\rho|<1$, then standard calculations show $\lim _{m \rightarrow \pm \infty} f(m)=\infty$, so by Proposition 4.2 there must be a critical moment both in $(-\infty, 0]$ and in $[1, \infty)$. In order to find the critical moments, we have to solve the equation

$$
\frac{R_{1}}{\nu}-\rho m=\sqrt{m^{2}-m}, \quad m \in \mathbb{R} \backslash(0,1) .
$$

Squaring both sides shows that a critical moment $m$ has to satisfy

$$
p(m)=m^{2}\left(1-\rho^{2}\right)+\left(2 \frac{R_{1}}{\nu} \rho-1\right) m-\frac{R_{1}^{2}}{\nu^{2}}=0 .
$$

If $|\rho|<1$, then $p$ is a convex parabola with $p(0)=-\frac{R_{1}^{2}}{\nu^{2}} \leq 0$ and $p(1)=-\rho^{2}-\frac{R_{1}^{2}}{\nu^{2}}+2 \frac{R_{1}}{\nu} \rho \leq 0$, where the second inequality follows from the assumption that $R_{1} \geq \nu \rho$. Therefore, $p$ has two real roots

$$
m_{ \pm}=\frac{1-2 \frac{R_{1}}{\nu} \rho \pm \sqrt{\left(1-2 \frac{R_{1}}{\nu} \rho\right)^{2}+4\left(1-\rho^{2}\right) \frac{R_{1}^{2}}{\nu^{2}}}}{2\left(1-\rho^{2}\right)}
$$

with $m_{-} \leq 0$ and $m_{+} \geq 1$. It is directly verified that $m_{ \pm}$solves (21).

If $\rho=-1$, then $\lim _{m \rightarrow \infty} f(m)=-\frac{1}{2}<0$. Therefore, $\frac{R_{1}}{\nu} \geq f(m)$ for all $m \geq 1$, so that $m_{+}=\infty$. Since $\lim _{m \rightarrow-\infty} f(m)=\infty$, there will be a critical moment in $(-\infty, 0]$ and it is given by the single root of $p$ :

$$
m_{-}=\frac{R_{1}^{2}}{-2 R_{1} \nu-\nu^{2}} \leq 0 .
$$

Similarly, if $\rho=1$, then $\lim _{m \rightarrow-\infty} f(m)=\frac{1}{2}$. Since we assume $R_{1} \geq \nu$ in this case, we have in particular $\frac{R_{1}}{\nu} \geq f(m)$ for all $m \leq 0$, so that $m_{-}=-\infty$. Since $\lim _{m \rightarrow \infty} f(m)=\infty$, there will be a critical moment in $[1, \infty)$ and it is given by the single root of $p$ :

$$
m_{+}=\frac{R_{1}^{2}}{2 R_{1} \nu-\nu^{2}} \geq 1
$$

where the inequality follows from the assumption $R_{1} \geq \rho \nu=\nu$. 


\section{A.6 Proof of Proposition 4.5}

1. Solving (11) gives

$$
\begin{aligned}
S_{T} & =S_{t} \exp \left\{-\int_{t}^{T} \sigma_{s} \mathrm{~d} W_{s}-\frac{1}{2} \int_{t}^{T} \sigma_{s}^{2} \mathrm{~d} s\right\} \\
& =S_{t} \exp \left\{-\frac{1}{\nu}\left(\sigma_{T}-\sigma_{t}-\int_{t}^{T}\left(R_{0}+R_{1} \sigma_{s}\right)\left(R_{2}-\sigma_{s}\right) \mathrm{d} s\right)-\frac{1}{2} \int_{t}^{T} \sigma_{s}^{2} \mathrm{~d} s\right\} \\
& =S_{t} \exp \left\{-\frac{1}{\nu}\left(\sigma_{T}-\sigma_{t}-R_{0} R_{2}(T-t)-\left(R_{1} R_{2}-R_{0}\right) \int_{t}^{T} \sigma_{s} \mathrm{~d} s\right)-\left(\frac{1}{2}+\frac{R_{1}}{\nu}\right) \int_{t}^{T} \sigma_{s}^{2} \mathrm{~d} s\right\} \\
& \leq S_{t} \exp \left\{\frac{\sigma_{t}}{\nu}+\frac{R_{0} R_{2}}{\nu}(T-t)\right\} .
\end{aligned}
$$

2. Similarly as in the first part, solving (11) gives

$$
\begin{aligned}
S_{T} & =S_{t} \exp \left\{\int_{t}^{T} \sigma_{s} \mathrm{~d} W_{s}-\frac{1}{2} \int_{t}^{T} \sigma_{s}^{2} \mathrm{~d} s\right\} \\
& =S_{t} \exp \left\{\frac{1}{\nu}\left(\sigma_{T}-\sigma_{t}-R_{0} R_{2}(T-t)-\left(R_{1} R_{2}-R_{0}\right) \int_{t}^{T} \sigma_{s} \mathrm{~d} s\right)-\left(\frac{1}{2}-\frac{R_{1}}{\nu}\right) \int_{t}^{T} \sigma_{s}^{2} \mathrm{~d} s\right\} \\
& \geq S_{t} \exp \left\{-\frac{\sigma_{t}}{\nu}-\frac{R_{0} R_{2}}{\nu}(T-t)\right\} .
\end{aligned}
$$

\section{A.7 Proof of Proposition 5.1}

The dynamics of $X_{t}:=z \sigma_{t}$ becomes

$$
\begin{aligned}
\mathrm{d} X_{t} & =\left(R_{0}+R_{1} \sigma_{t}\right)\left(R_{2} z-X_{t}\right) \mathrm{d} t+\nu X_{t} \mathrm{~d} W_{t} \\
& =\left(R_{0}+\nu X_{t}\right)\left(\frac{R_{2} R_{1}}{\nu}-X_{t}\right) \mathrm{d} t+\nu X_{t} \mathrm{~d} W_{t} \\
& =b\left(X_{t}\right) \mathrm{d} t+\nu X_{t} \mathrm{~d} W_{t},
\end{aligned}
$$

where we defined the function $b(x)=\left(R_{0}+\nu x\right)\left(\frac{R_{2} R_{1}}{\nu}-x\right)$. The dynamics of $\mathrm{e}^{y t}$ becomes

$$
\mathrm{de}^{y_{t}}=X_{t} \mathrm{e}^{y_{t}} \mathrm{~d} W_{t} .
$$

We conclude by Theorem 2.4(i) in Lions and Musiela (2007) that $\mathrm{e}^{y_{t}}$ is a $\mathbb{Q}$-martingale, since

$$
\lim _{x \rightarrow+\infty} \frac{b(x)+\nu x^{2}}{x}=R_{2} R_{1}-R_{0}<\infty .
$$

\section{A.8 Proof of Lemma 5.2}

It is well known that $\operatorname{dim}\left(\operatorname{Pol}_{m}\left(\mathbb{R}^{n}\right)\right)=\left(\begin{array}{c}m+n \\ n\end{array}\right)$. As a consequence, the dimension of the set of polynomials in $\mathbb{R}^{n}$ with total degree exactly equal to $k \in \mathbb{N}$ is

$$
\left(\begin{array}{c}
k+n \\
n
\end{array}\right)-\left(\begin{array}{c}
k-1+n \\
n
\end{array}\right)=k+1
$$


where we define $\left(\begin{array}{c}n-1 \\ n\end{array}\right)=0$. We therefore get

$$
\begin{aligned}
\operatorname{dim}\left(P_{m}\right) & =\sum_{k=0}^{m}(k+1)(1+2(m-k)) \\
& =(2 m+1)(m+1)+(2 m-1) \sum_{k=0}^{m} k-2 \sum_{k=0}^{m} k^{2} \\
& =(2 m+1)(m+1)+\frac{1}{2} m(m+1)(2 m-1)-\frac{1}{3} m(m+1)(2 m+1) \\
& =\frac{1}{3} m^{3}+\frac{3}{2} m^{2}+\frac{13}{6} m+1
\end{aligned}
$$

\section{A.9 Proof of Proposition 5.3}

Without loss of generality, we can use a monomial basis for $P_{m}$. A generic element in this basis can be represented as $x^{\alpha} y^{\beta} z^{\gamma}$, with $\alpha, \beta, \gamma \in \mathbb{N}, \alpha+\beta \leq m$ and $\gamma \leq 2(m-\alpha-\beta)$. Applying the $\mathbb{Q}^{z}$-generator $\mathcal{G}$ of $\left(x_{t}, y_{t}, \sigma_{t}\right)$ to this monomial gives

$$
\begin{aligned}
\mathcal{G} x^{\alpha} y^{\beta} z^{\gamma}= & \alpha\left(z \rho-\frac{1}{2}\right) x^{\alpha-1} y^{\beta} z^{\gamma+2}+\beta \frac{1}{2} z^{2} x^{\alpha} y^{\beta-1} z^{\gamma+2}+\gamma R_{0} R_{2} x^{\alpha} y^{\beta} z^{\gamma-1}+\gamma\left(R_{1} R_{2}-R_{0}\right) x^{\alpha} y^{\beta} z^{\gamma} \\
& +\frac{1}{2} \alpha(\alpha-1) x^{\alpha-2} y^{\beta} z^{\gamma+2}+\frac{1}{2} \beta(\beta-1) z^{2} x^{\alpha} y^{\beta-2} z^{\gamma+2}+\frac{1}{2} \gamma(\gamma-1) \nu^{2} x^{\alpha} y^{\beta} z^{\gamma} \\
& +\alpha \beta z \rho x^{\alpha-1} y^{\beta-1} z^{\gamma+2}+\alpha \gamma \nu \rho x^{\alpha-1} y^{\beta} z^{\gamma+1}+\beta \gamma z \nu x^{\alpha} y^{\beta-1} z^{\gamma+1}
\end{aligned}
$$

It is readily verified by inspecting each of the above monomials that $\mathcal{G} x^{\alpha} y^{\beta} z^{\gamma} \in P_{m}$.

\section{A.10 Proof of Proposition 6.1}

The optimization problem in (14) is a convex quadratic programming problem. The first order conditions become

$$
2 \int_{\mathbb{R}^{2}}\left(e^{-y} F\left(e^{x}\right)-c^{\top} B_{n}(x, y)\right) b_{i}(x, y) w(x, y) \mathrm{d} x \mathrm{~d} y=0, \quad i=1, \ldots, N_{n} .
$$

Re-arranging terms we get

$$
\sum_{j=1}^{N_{n}} \int_{\mathbb{R}^{2}} c_{j} b_{j}(x, y) b_{i}(x, y) w(x, y) \mathrm{d} x \mathrm{~d} y=\int_{\mathbb{R}^{2}} e^{-y} F\left(e^{x}\right) b_{i}(x, y) w(x, y) \mathrm{d} x \mathrm{~d} y .
$$

In matrix notation this becomes

$$
D c=f .
$$

Remark that the matrix $D$ positive-definite by construction and therefore invertible. 


\section{A.11 Proof of Proposition 6.2}

To lighten the notation, we suppress the superscript $(k)$ throughout the proof. Using the identity $x \phi(x)=-\phi^{\prime}(x)$ and integrating by parts gives

$$
\begin{aligned}
I_{n} & =\int_{\mathbb{R}}\left(\mathrm{e}^{x}-K\right)^{+} x^{n} \phi_{m, v}(x) \mathrm{d} x \\
& =\int_{\mathbb{R}}\left(\mathrm{e}^{m+\sqrt{v} x}-K\right)^{+}(m+\sqrt{v} x)^{n} \phi(x) \mathrm{d} x \\
& =\int_{-\xi}^{\infty}\left(\mathrm{e}^{m+\sqrt{v} x}-K\right)(m+\sqrt{v} x)^{n} \phi(x) \mathrm{d} x \\
& =m I_{n-1}+\sqrt{v} \int_{-\xi}^{\infty}\left(\mathrm{e}^{m+\sqrt{v} x}-K\right)(m+\sqrt{v} x)^{n-1} x \phi(x) \mathrm{d} x \\
& =m I_{n-1}-\sqrt{v} \int_{-\xi}^{\infty}\left(\mathrm{e}^{m+\sqrt{v} x}-K\right)(m+\sqrt{v} x)^{n-1} \phi^{\prime}(x) \mathrm{d} x \\
& =m I_{n-1}-\sqrt{v}\left[-\sqrt{v}(n-1) I_{n-2}-\sqrt{v} \int_{-\xi}^{\infty} \mathrm{e}^{m+\sqrt{v} x}(m+\sqrt{v} x)^{n-1} \phi^{\prime}(x) \mathrm{d} x\right] \\
& =(m+v) I_{n-1}+v(n-1) I_{n-2}+K v \int_{-\xi}^{\infty}(m+\sqrt{v} x)^{n-1} \phi(x) \mathrm{d} x .
\end{aligned}
$$

Define $J_{n}=\int_{-\xi}^{\infty}(m+\sqrt{v} x)^{n} \phi(x) \mathrm{d} x$. Similarly as for $I_{n}$, we use integration by parts to derive the following recursion for $J_{n}$

$$
\begin{aligned}
J_{n} & =m J_{n-1}-\sqrt{v} \int_{-\xi}^{\infty}(m+\sqrt{v} x)^{n-1} \phi^{\prime}(x) \mathrm{d} x \\
& =m J_{n-1}-\sqrt{v}\left[-(m-\sqrt{v} \xi)^{n-1} \phi(-\xi)-(n-1) \sqrt{v} \int_{-\xi}^{\infty}(m+\sqrt{v} x)^{n-2} \phi^{\prime}(x) \mathrm{d} x\right] \\
& =m J_{n-1}+v(n-1) J_{n-2}+\sqrt{v}(\log (K))^{n-1} \phi(\xi) .
\end{aligned}
$$

For the starting values of the recursion, we have

$$
\begin{aligned}
& J_{0}=\int_{-x i}^{\infty} \phi(x) \mathrm{d} x=\Phi(\xi), \\
& I_{0}=\mathrm{e}^{m+\frac{1}{2} v} \Phi(\xi+\sqrt{v})-K \Phi(\xi) .
\end{aligned}
$$

We omit the full derivation of $I_{0}$ since it is very similar to computing the price of a European call option in the Black-Scholes model.

\section{B Auxiliary results}

\section{B.1 Lower bound on first moment of steady-state density}

Suppose the assumptions of Proposition 3.1 are satisfied so that a non-trivial steady-state distribution exists, and suppose furthermore that $R_{1}>0$. We introduce for simplicity the following notation

$$
\alpha=-2 \frac{R_{0} R_{2}}{\nu^{2}}, \quad \beta--2 \frac{R_{1}}{\nu^{2}} .
$$


The first moment of the steady-state density then becomes

$$
\begin{aligned}
\int_{0}^{\infty} x \pi(x) \mathrm{d} x & =\frac{\int_{0}^{\infty} x^{\xi} \exp \left\{\alpha \frac{1}{x}+\beta x\right\} \mathrm{d} x}{\int_{0}^{\infty} x^{\xi-1} \exp \left\{\alpha \frac{1}{x}+\beta x\right\} \mathrm{d} x} \\
& =\frac{\int_{0}^{\infty} x^{\xi} \exp \left\{\alpha \frac{1}{x}\right\} \mathrm{d}\left(\frac{\exp \{\beta x\}}{\beta}\right)}{\int_{0}^{\infty} x^{\xi-1} \exp \left\{\alpha \frac{1}{x}+\beta x\right\} \mathrm{d} x} \\
& =-\frac{\xi}{\beta}+\frac{\alpha}{\beta} \frac{\int_{0}^{\infty} x^{\xi-2} \exp \left\{\alpha \frac{1}{x}+\beta x\right\} \mathrm{d} x}{\int_{0}^{\infty} x^{\xi-1} \exp \left\{\alpha \frac{1}{x}+\beta x\right\} \mathrm{d} x} \\
& =-\frac{\xi}{\beta}+\frac{\alpha}{\beta} \int_{0}^{\infty} x^{-1} \pi(x) \mathrm{d} x
\end{aligned}
$$

where we have used integration by parts on the integral in the numerator. Remark now that $x \mapsto 1 / x$ is strictly convex for $x>0$, so we have by Jensen's inequality

$$
\int_{0}^{\infty} x \pi(x) \mathrm{d} x \geq-\frac{\xi}{\beta}+\frac{\alpha}{\beta}\left(\int_{0}^{\infty} x \pi(x) \mathrm{d} x\right)^{-1} .
$$

Remark that the inequality is strict if and only if $R_{0} \neq 0$. If we denote $\mu=\int_{0}^{\infty} x \pi(x) \mathrm{d} x$, we obtain the following quadratic inequality for $\mu$

$$
\mu^{2}+\frac{\xi}{\beta} \mu-\frac{\alpha}{\beta} \geq 0 .
$$

By solving the roots of the parabola, we see that the above inequality can only be true if

$$
\mu \geq \frac{1}{2}\left(-\frac{\xi}{\beta}+\sqrt{\frac{\xi^{2}}{\beta^{2}}+4 \frac{\alpha}{\beta}}\right) .
$$

\section{B.2 Moments of the Gaussian distribution}

Suppose we want to compute all moments of a univariate Gaussian distribution with mean $\mu \in \mathbb{R}$ and variance $\sigma^{2}>0$. Consider the Gaussian process $X_{t}$ defined through the following SDE

$$
\mathrm{d} X_{t}=\mu \mathrm{d} t+\sigma \mathrm{d} W_{t}, \quad X_{0}=0
$$

where $W_{t}$ is a standard Brownian motion. The solution $X_{1}$ at time 1 has a Gaussian distribution with mean $\mu$ and variance $\sigma^{2}$. Applying the infinitesimal generator $\mathcal{G}$ of $X_{t}$ to a monomial $x^{n}$ gives

$$
\mathcal{G} x^{n}=n x^{n-1} \mu+\frac{1}{2} n(n-1) x^{n-2} \sigma^{2} .
$$

Therefore, if we define $G_{n} \in \mathbb{R}^{(n+1) \times(n+1)}$ as

$$
G_{n}=\left(\begin{array}{cccccc}
0 & & & & & \\
\mu & 0 & & & & \\
\sigma^{2} & 2 \mu & 0 & & & \\
0 & 3 \sigma^{2} & 3 \mu & 0 & & \\
\vdots & \ddots & \ddots & \ddots & \ddots & \\
0 & \cdots & 0 & \frac{\sigma^{2} n(n-1)}{2} & n \mu & 0
\end{array}\right),
$$


then we can write

$$
\mathcal{G}\left(\begin{array}{lllll}
1 & x & x^{2} & \cdots & x^{n}
\end{array}\right)^{\top}=G_{n}\left(\begin{array}{lllll}
1 & x & x^{2} & \cdots & x^{n}
\end{array}\right)^{\top}
$$

By definition of the generator, we get the following simple formula for the Gaussian moments

$$
\left.\mathbb{E}_{0}\left[\begin{array}{lllll}
1 & X_{1} & X_{1}^{2} & \cdots & X_{1}^{n}
\end{array}\right)^{\top}\right]=\mathrm{e}^{G_{n}}\left(\begin{array}{lllll}
1 & 0 & 0 & \cdots & 0
\end{array}\right)^{\top} .
$$




\section{References}

Ackerer, D. and D. Filipović (2016). Linear credit risk models. arXiv preprint arXiv:1605.07419.

Ackerer, D. and D. Filipović (2019). Option pricing with orthogonal polynomial expansions. Mathematical Finance, Forthcoming.

Ackerer, D., D. Filipović, and S. Pulido (2018). The Jacobi stochastic volatility model. Finance and Stochastics 22(3), 667-700.

Andersen, L. B. and V. V. Piterbarg (2007). Moment explosions in stochastic volatility models. Finance and Stochastics 11(1), 29-50.

Andersen, T. G., T. Bollerslev, F. X. Diebold, and H. Ebens (2001). The distribution of realized stock return volatility. Journal of Financial Economics 61(1), 43-76.

Andersen, T. G., T. Bollerslev, F. X. Diebold, and P. Labys (2001). The distribution of realized exchange rate volatility. Journal of the American Statistical Association 96(453), 42-55.

Bakshi, G., N. Ju, and H. Ou-Yang (2006). Estimation of continuous-time models with an application to equity volatility dynamics. Journal of Financial Economics 82(1), 227-249.

Barndorff-Nielsen, O. E. (1997). Normal inverse Gaussian distributions and stochastic volatility modelling. Scandinavian Journal of statistics 24(1), 1-13.

Barone-Adesi, G., H. Rasmussen, and C. Ravanelli (2005). An option pricing formula for the GARCH diffusion model. Computational Statistics 85 Data Analysis 49(2), 287-310.

Christoffersen, P., K. Jacobs, and K. Mimouni (2010). Volatility dynamics for the S\&P500: evidence from realized volatility, daily returns, and option prices. The Review of Financial Studies 23(8), 3141-3189.

Duffie, D., D. Filipović, W. Schachermayer, et al. (2003). Affine processes and applications in finance. The Annals of Applied Probability 13(3), 984-1053.

Eberlein, E. (2001). Application of generalized hyperbolic Lévy motions to finance. In Lévy processes, pp. 319-336. Springer.

Eberlein, E. and K. Prause (2002). The generalized hyperbolic model: financial derivatives and risk measures. In Mathematical Finance - Bachelier Congress 2000, pp. 245-267. Springer.

Ewald, C.-O. and Z. Yang (2007). Geometric mean reversion: formulas for the equilibrium density and analytic moment matching. Available at SSRN 999561.

Filipović, D., E. Gourier, and L. Mancini (2016). Quadratic variance swap models. Journal of Financial Economics 119(1), 44-68.

Filipović, D. and M. Larsson (2016). Polynomial diffusions and applications in finance. Finance and Stochastics 20(4), 931-972.

Filipović, D. and S. Willems (2017). A term structure model for dividends and interest rates. Working Paper. 
Gander, M. P. and D. A. Stephens (2007). Stochastic volatility modelling in continuous time with general marginal distributions: Inference, prediction and model selection. Journal of Statistical Planning and Inference 137(10), 3068-3081.

Hagan, P. S., D. Kumar, A. S. Lesniewski, and D. E. Woodward (2002). Managing smile risk. The Best of Wilmott 1, 249-296.

Heston, S. L. (1993). A closed-form solution for options with stochastic volatility with applications to bond and currency options. The Review of Financial Studies 6(2), 327-343.

Hull, J. and A. White (1987). The pricing of options on assets with stochastic volatilities. The Journal of Finance 42(2), 281-300.

Ikeda, N. and S. Watanabe (1989). Stochastic Differential Equations and Diffusion Processes (2nd ed.), Volume 24. Elsevier.

Jäckel, P. (2005). A note on multivariate gauss-hermite quadrature. Technical report.

Jorgensen, B. (1982). Statistical Properties of the Generalized Inverse Gaussian Distribution, Volume 9. Springer Science \& Business Media.

Kahl, C. and P. Jäckel (2006). Fast strong approximation monte carlo schemes for stochastic volatility models. Quantitative Finance 6(6), 513-536.

Karasinski, P. and A. Sepp (2012). Beta stochastic volatility model. Risk, 66-71.

Karatzas, I. and S. Shreve (1991). Brownian Motion and Stochastic Calculus (2nd ed.). SpringerVerlag.

Keller-Ressel, M. (2011). Moment explosions and long-term behavior of affine stochastic volatility models. Mathematical Finance: An International Journal of Mathematics, Statistics and Financial Economics 21(1), 73-98.

Kloeden, P. E. and E. Platen (1995). Numerical Solution of Stochastic Differential Equations, Volume 23. Springer Science \& Business Media.

Lee, G., Z. Zhu, et al. (2016). Switching to non-affine stochastic volatility: A closed-form expansion for the Inverse Gamma model. Technical report.

Lee, R. W. (2004). The moment formula for implied volatility at extreme strikes. Mathematical Finance 14(3), 469-480.

Lewis, A. L. (2000). Option Valuation Under Stochastic Volatility: With Mathematica Code. Finance Press.

Lewis, A. L. (2019). Exact solutions for a GBM-type stochastic volatility model having a stationary distribution. arXiv preprint arXiv:1809.08635.

Lions, P.-L. and M. Musiela (2007). Correlations and bounds for stochastic volatility models. In Annales de l'Institut Henri Poincare (C) Non Linear Analysis, Volume 24, pp. 1-16. Elsevier.

Merton, R. C. (1975). An asymptotic theory of growth under uncertainty. The Review of Economic Studies 42(3), 375-393. 
Nelson, D. B. (1990). ARCH models as diffusion approximations. Journal of Econometrics 45(12), 7-38.

Pagès, G. and J. Printems (2003). Optimal quadratic quantization for numerics: the Gaussian case. Monte Carlo Methods and Applications 9(2), 135-165.

Sepp, A. (2014). Empirical calibration and minimum-variance delta under log-normal stochastic volatility dynamics. Available at SSRN 2387845.

Sepp, A. (2016). Log-normal stochastic volatility model: Affine decomposition of moment generating function and pricing of vanilla options.

Tuckwell, H. C. and J. A. Koziol (1987). Logistic population growth under random dispersal. Bulletin of Mathematical Biology 49(4), 495-506.

Willems, S. (2019). Asian option pricing with orthogonal polynomials. Quantitative Finance 19(4), 605-618. 